\title{
Effekte elektromagnetischer Felder auf biologische Systeme und Expressionsmuster von Wachstumsfaktoren. Ein Review
}

\author{
Gerhard W. Ruoff \\ Sevelen, Schweiz
}

D as Leben auf unserer Erde entwickelte sich unter dem Einfluss eines sich langzeitlich ändernden natürlichen Magnetfeldes, dem sogenannten Erdmagnetfeld. Das sich langzeitlich ändernde, natürliche Magnetfeld der Erde besitzt derzeit in unseren Breitengraden eine magnetische Flussdichte von im Mittel $50 \mu$ T. Dass dieses magnetische Feld das Leben auf der Erde beeinflusst, geht eindrucksvoll aus einer in der tschechischen Republik erstellten Filmdokumentation zur Entwicklung von Hühner-Embryonen hervor [1]. Hühner, welche während ihrer Entwicklung von diesem natürlichen Feld abgeschirmt worden waren, waren gegenüber ihren, unter natürlichen Feldbedingungen aufgezogenen, Artgenossen deutlich in ihren Bewegungen eingeschränkt (Abb. 1). Offensichtlich hat sich das Leben, zumindest während der Entwicklungsphase eines Organismus, an die Existenz dieses Feldes angepasst. Massnahmen zur Erhaltung bzw. Wiederherstellung der Gesundheit mittels elektromagnetischer Felder scheinen auf eine Unterstützung natürlich vorgegebener Mechanismen zur Selbstregulation hin ausgerichtet zu sein.

Entsprechend den Gleichungen des schottischen Physikers JAMEs CLERK MAXWELL (1831-1879) besitzen elektromagnetische Felder einen magnetischen und einen elektrischen Vektor. Elektrische und magnetische Felder kommen normalerweise nicht getrennt voneinander vor und werden daher vereinfachend als elektromagnetische Felder bezeichnet ${ }^{1}$. Sie unterscheiden sich u.a. in Abhängigkeit von der Geschwindig-

1 Beide Feldarten bedingen sich im Falle einer zeitlichen Veränderung insofern gegenseitig, als ein sich änderndes elektrisches Feld ein magnetisches und ein sich erzeugt.

Hintergrund: Die Wirkung elektromagnetischer Felder auf biologische Systeme ist bislang nur unvollständig erforscht und ein beliebtes Objekt für Spekulationen aller Art seitens der Hersteller und Vertreiber von Elektromagnetfeldsystemen. Zielsetzung: Erstellung einer Übersicht zum derzeitigen wissenschaftlichen Kenntnisstand zur Wirkung der in den heute gebräuchlichen Therapiesystemen verwendeten elektromagnetischen Signale, beispielhaft dargestellt anhand ihrer Wirkung auf die Expressionsmuster von Wachstumsfaktoren. Methodik: Systematische Analyse wissenschaftlicher Publikationen (1990-2008), zugänglich über die elektronischen Datenbanken PUBMED und DIMDI. Ergebnisse und Schlussfolgerungen: Ein signifikanter Einfluss elektromagnetischer Felder ist beschrieben beim Epidermalen Wachstumsfaktor (EGF), dem insulinähnlichen Wachstumsfaktor 2 (IGF-2), dem Fibroblasten-Wachstumsfaktor 2 (FGF-2), dem Nerven-Wachstumsfaktor (NGF), dem transformierenden Wachstumsfaktor Beta (TGF-ß) und den zu dieser Gruppe zählenden Knochenmorphogenese-Proteinen 2 und 4 (BMP-2, -4). Insgesamt zeigt sich nicht nur ein auf molekularer Ebene äusserst adaptiver, vielfältiger und individueller Einfluss elektromagnetischer Signale auf die Expression von Wachstumsfaktoren. Elektromagnetische Signale aktivieren offensichtlich völlig unterschiedliche molekulare Prozesse.

Schlüsselwörter: Elektromagnetische Stimulation, Elektromagnetisches Signal, Wachstumsfaktor, Elektromagnetfeld-Therapie

\section{Effects of Electromagnetic Fields on Biological Systems and Expression Patterns of Growth Factors. A Review}

Background: To date, the efficacy of electromagnetic fields on biological systems has not been well investigated and is a popular topic for all kinds of speculation among manufacturers and distributors of electromagnetic systems. Objective: To provide an overview of current scientific knowledge on the efficacy of electromagnetic signals used in common therapeutic systems, as illustrated by the example of expression patterns of growth factors. Method: Systematic analysis of scientific literature since 1990, available in the databases PUBMED and DIMDI. Results and Conclusion: A significant influence of electromagnetic fields appeared on the Epidermal Growth Factor (EGF), the Insulin-like Growth Factor 2 (IGF-2), the Fibroblast Growth Factor 2 (FGF-2), the Nerve Growth Factor (FGF), the Transforming Growth Factor Beta (TGF- $\beta$ ) and the Bone Morphogenetic Proteins 2 and $4(B M P-2,-4)$ belonging to the same group of growth factors. Altogether, not only a clearly adaptive, manifold and individual influence of electromagnetic signals on the expression of growth factors has been noticed, it seems that electromagnetic signals may thereby activate completely different molecular processes.

Key words: Electromagnetic stimulation, electromagnetic signal, growth factor, electromagnetic therapy

keit der zeitlichen Veränderung, in ihrer Fähigkeit zur Materialdurchdringung. Im Gegensatz zu einem elektrischen Feld durchdringt ein magnetisches Feld alle Zellen des von ihm beaufschlagten Organismus vollständig und nahezu verlustfrei, d.h. dass dabei nur relativ geringe Mengen an Energie übertragen werden. Die damit mögliche nicht invasive Anwendung stellt für den Arzt eine einfache und für den $\mathrm{Pa}$ tienten eine angenehme Therapieoption dar. Ein weiterer Vorteil ist, wie weiter unten gezeigt, ihre Wirksamkeit bereits bei Energiedichten von geringem energetischem Niveau. So ist z.B. bei einem elektromagnetischen Feld die zur Induktion der Synthese des Stressproteins HSP70 notwendige Energiedichte mit $2,6 \times 10^{-7} \mathrm{~J} / \mathrm{m}^{3} \mathrm{im}$ Vergleich zu 2,3 $\times 10^{7} \mathrm{~J} / \mathrm{m}^{3}$ um den Faktor 14 geringer als bei Hitzeschock [2].

\section{Das elektromagnetische Feld}

Die heute in der Elektromagnetfeld-Therapie üblichen elektromagnetischen Fel- 

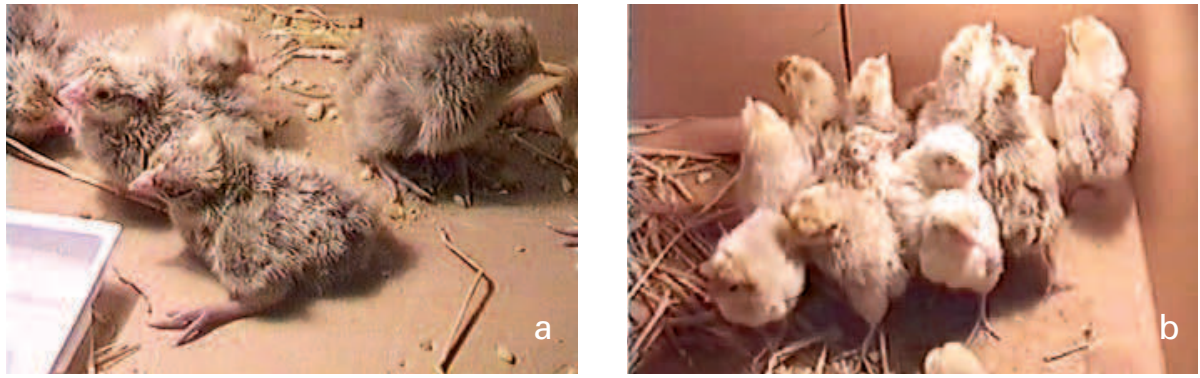

Abb. 1. Aufzucht von Küken ohne (a) und unter dem Einfluss des Geomagnetischen Feldes (b) Die Bilder stammen aus einer mit freundlicher Genehmigung des Autors [1] zur Verfügung gestellten Videoaufzeichnung.

der lassen sich beispielsweise in Anlehnung an MARKov [3] grob einteilen in:

- Permanente Magnetfelder, erzeugt durch einen Permanentmagneten bzw. durch eine von Gleichstrom durchflossene Spule.

Elektromagnetische Felder mit niederfrequenten, sinusförmig verlaufenden Intensitätsvariationen.

- Sinus-, rechteck-, trapez-, sägezahnförmig oder komplex modulierte auch in Bursts - gepulste elektromagnetische Felder.

- Sehr hochfrequente Felder mit Wellenlängen im Millimeterbereich.

- Gepulste Felder mit Radiofrequenzen.

- Kurze Signale mit starken Magnetfeldern für die transkraniale Stimulation.

Letztendlich unterscheiden sich diese Felder in ihren, z.B. durch Analyse der spektralen Zusammensetzung charakterisierbaren, zeitlichen Intensitätsverläufen. Bislang ist noch offen, in wie weit sich die induzierten biologischen, auf atomistisch-molekularen Vorgängen basierenden Wirkungen den spektralen Komponenten zuordnen lassen. Die durch unterschiedliche Systeme erzielten elektromagnetisch induzierten Wirkungen dürfen keinesfalls pauschal auf elektromagnetische Applikationen, insbesondere die Elektromagnetfeldtherapie, als solche übertragen werden.

Darüber hinaus beschreibt nahezu jeder Hersteller sein elektromagnetisches System nach eigenen Kriterien, so dass in den Publikationen der damit durchgeführten Studien die das applizierte elektromagnetische Feld ausreichend charakterisierenden Daten selten zu finden sind. Doch selbst wenn diese Details hinlänglich bekannt sind, ent- sprechen die bei der Exposition ausserhalb des Organismus gemessenen Werte nicht zwingend jenen am Gewebe bzw. Organ im Körper. Unabhängig davon ist in der Regel nicht bestimmbar, welchen Beitrag das geomagnetische Feld der Erde zum Ergebnis beigetragen hat.

Ein Teil der heute in der Therapie noch verwendeten elektromagnetischen Systeme arbeitet mit einer Frequenz von $50 \mathrm{~Hz}$ bzw. $60 \mathrm{~Hz}$ auf der Basis sinusförmiger Signale oder Rechtecksignale. Die häufige Verwendung von 50- oder 60-Hz-Sinus- oder Rechtecksignalen über viele Jahre sollte allerdings nicht als Hinweis auf deren besondere Wirksamkeit interpretiert werden. Diese Frequenzen und Signalformen verdanken ihre Beliebtheit ihrer bevorzugten Verwendung in elektrotechnischen Laboratorien aufgrund ihrer leichten technischen Umsetzbarkeit und Praktikabilität. Ausserdem sind Rechtecksignale mit - zum Erreichen der steilen Flanken notwendigen - hohen Frequenzanteilen in der belebten Natur unbekannt.

Die in der Elektromagnetfeldtherapie vermutlich bekannteste Signalform geht zurück auf das zur Beschleunigung von Frakturheilungen von BASSETT 1974 beschriebene sog. Sägezahn-Signal. Eine Signalform, welche aufgrund ihrer raschen Intensitätswechsel in Geweben relativ hohe Stromstärken erzeugen kann heute verwendete Signale wurde [4].

In den moderneren therapeutischen Systemen finden sich zunehmend gepulste monopolare bzw. bipolare Signalformen, basierend auf einfachen, sinusförmigen Signalen bis hin zu Signalformen mit den einem Rechtecksignal ähnelnden, jedoch aus vielen Einzelsignalen bestehenden sog. Bursts. und damit zum Vorreiter für viele,
BLANK und Goodman untersuchten, welchen Einfluss die Frequenz bei einem elektromagnetischen Feld auf die Biosynthese und enzymatische Reaktionen hat [5]. Als optimale Frequenz des elektrischen Feldes bei der In-vivoBiosynthese langsamer Muskelfasern von Säugetieren fanden sie $20 \mathrm{~Hz}$, wohingegen bei der Reaktionsrate der protonenpumpenden ATP-Synthase $\left(\mathrm{F}_{1} \mathrm{~F}_{0}\right.$ ATPase) bei der elektrischen Feldstärke von 2,3 V/cm $1 \mathrm{kHz}$ bzw. bei der höheren elektrischen Feldstärke von 3,6 V/cm $30 \mathrm{~Hz}$ und beim Enzym Cytochrom-Oxidase $800 \mathrm{~Hz}$ ermittelt wurden. Demzufolge lassen sich durch unterschiedliche elektromagnetische Stimulationen unterschiedliche biologische Wirkungen initiieren und Elektromagnetfeldsysteme mit einem spektral breit zusammengesetzten Signal lassen breiter gestreute Wirkungen erwarten.

\section{Praktische Anwendung}

Für die Heimanwendung und auch in der medizinischen Praxis werden zur Ganzkörperbehandlung vorzugsweise flexible sog. Matten mit darin eingearbeiteten, stromdurchflossenen Spulen zur Erzeugung des elektromagnetischen Feldes verwendet. Neben den Matten finden sich von Spulen durchflossene Röhren, welche den zu behandelnden Körperteil umschliessen. Für speziell lokale Applikationen und mit höheren Intensitäten gibt es kleinere Matten, sog. Kissen, und die aus einer einzelnen Spule bestehenden, rechteckig oder stabförmig aussehenden „Intensiv-Applikatoren“. In der medizinischen Praxis sind speziell dafür konzipierte, stationäre Systeme im Einsatz. Unabhängig davon ist das elektromagnetische Feld, je nach Anordnung der Spulen, homogen oder inhomogen. In einem homogenen Feld sind die resultierenden elektrischen/magnetischen Kraftwirkungen auf einen elektrischen/magnetischen Dipol allerdings gleich Null, während bei einem inhomogenen Feld von der jeweiligen Polarität abhängige, zusätzliche Anziehungen und Abstossungen in den inhomogenen Feldbereich hinein bzw. daraus heraus möglich sind.

Ungeachtet der unterschiedlich eingesetzten Elektro-Magnetfeldsysteme und damit auch der unterschiedlichen Stimulationscharakteristika - finden 
sich in der Literatur zahlreiche, statistisch abgesicherte Hinweise und entsprechend den Kriterien der evidenzbasierten Medizin beurteilte effiziente, von der Behandlungsdauer abhängige therapeutische Effekte in der Knochenund Wundheilung sowie der Schmerzbehandlung bei degenerativen Gelenkserkrankungen [6,7]. Weitere Anwendungsmöglichkeiten sind nicht auszuschliessen. Hierzu fehlt es jedoch zu den einzelnen Indikationen an einer ausreichend hohen Anzahl an vergleichbaren Studien mit auch akzeptablem Design. Dennoch ist diese Art einer therapeutischen Intervention selbst bei den bereits belegten Indikationen trotz ihrer Vorteile noch weit von einer medizinischen Anerkennung als Standardmethode entfernt. Dieses liegt sicherlich daran, dass der biophysikalische Mechanismus von schwachen elektrischen, magnetischen oder elektromagnetischen Feldern auf die verschiedenen biologischen Systeme noch nicht hinreichend wissenschaftlich untersucht ist.

Viele Anbieter von elektromagnetischen Therapiesystemen und deren Protagonisten nutzen geschickt diese Lücke aus, bewerben nach dem Motto: „Was nicht bewiesen ist, kann auch nicht widerlegt werden “ ihre Produkte munter mit „wissenschaftlichen Erkenntnissen“ beim fachlich unkundigen Publikum und schlagen dabei jeden seriös handelnden Naturwissenschaftler oder Mediziner in die Flucht.

\section{Trennung der Spreu vom Weizen}

Einer der wohl populärsten Irrtümer zur Wirkung elektromagnetischer Felder auf den Organismus ist vermutlich dessen vermeintlicher Einfluss auf das Membranpotential von Körperzellen. Bereits eine einfache Rechnung ergibt bei der Dicke einer Zellmembran von $7 \mathrm{~nm}$ und einer Potenzial-Differenz zwischen den beiden Oberflächen von $50 \mathrm{mV}$ eine elektrische Feldstärke von $7,14 \times$ $10^{6} \mathrm{~V} / \mathrm{m}$. Eine Grössenordnung, welche durch ein nicht invasives, von aussen auf den Körper einwirkendes, schwaches elektromagnetisches Feld nicht im entferntesten beeinflusst werden kann [8].

Ein elektromagnetisches Feld wird auch nicht die Synthese an AdenosinTri-Phosphat (ATP) zu einer höheren Leistung treiben, um dem einer Krank- heit vermeintlich zugrunde liegenden Energiemangel entgegenzuwirken. Nicht nur, dass den meisten Patienten eine Gewichtsreduktion empfohlen werden muss und bei einer Bildung von 51,4 Mol ATP aus nur $100 \mathrm{~g}$ Fett (Tristearin) der vermeintlich vorliegende Energiemangel nicht erklärbar ist. Der erwachsene Organismus enthält auch weniger als 0,1 Mol ATP, benötigt jedoch zur Deckung des Bedarfs an metabolischer Energie von täglich beispielsweise $1800 \mathrm{kcal}$ (7500 kJ) mehr als 200 Mol ATP. Damit ATP ständig, aber nur so schnell wie notwendig, gebildet wird, unterliegen die an einer ATP-Bildung beteiligten Stoffwechselaktivitäten einer streng koordinierten Kontrolle mit aufeinander abgestimmten Kontrollmechanismen der Glykogenolyse, der Glykolyse, dem Citratcyclus und der Geschwindigkeit der oxydativen Phosphorylierung. Es ist weder anhand der vielen daran beteiligten, biochemischen Mechanismen erklärbar, noch gibt es bisweilen ernst zu nehmende Hinweise darauf, dass ein dermassen komplexer Mechanismus alleine durch die Applikation eines elektromagnetischen Feldes beeinflussbar wäre.

Auch die Beeinflussung der Durchblutung in der Mikrozirkulation alleine durch ein magnetisches oder elektromagnetisches Feld ist noch nicht eindeutig bestätigt worden. McKaY et al. schliessen diesen Einfluss nach einer eingehenden Prüfung der Ergebnisse zahlreicher Human- und Tierstudien zwar nicht vollständig aus, sehen dieses jedoch dennoch als alles andere als erwiesen an [9]. SCHUHFRIED et al. konnten in ihrer Untersuchung an gesunden Probanden weder bei einem niedrig, noch hoch dosierten pulsierenden elektromagnetischen Feld einen signifikanten Einfluss auf die Temperatur und Mikrozirkulation in der Haut feststellen [10].

Über die den Wirkungen von elektrischen, magnetischen und elektromagnetischen Feldern zugrunde liegenden Mechanismen gibt es unter den ernst zu nehmenden Experten noch keine gesicherte einheitliche Aussage. Auf molekularer Ebene sind im Zusammenhang mit der Beeinflussung biologischer Systeme durch schwache elektromagnetische Felder aktuell in Fachkreisen u.a. ein parametrisches IonenResonanz-Modell, ein parametrisches
Ionen-Interferenz-Modell [11] sowie, ausgehend von Erkenntnissen über $\mathrm{Ca}^{2+}$-Signalwege, die Steuerung von Ionen und Substratwege durch Mikrovilli [12] in der Diskussion.

\section{Einfluss auf Wachstumsfaktoren}

In vorwiegend experimentellen Studien wird ein Einfluss schwacher elektromagnetischer Felder auf die als Wachstumsfaktoren bezeichnete, grosse Gruppe meist komplexer Proteine beschrieben: lokale Botenstoffe, welche bereits bei sehr niedrigen Konzentrationen wirksam sind und Wachstum, Vermehrung, Differenzierung oder das Überleben von Zellen in Geweben ermöglichen. Um einen Überblick über den aktuellen Stand der Erkenntnisse zum Einfluss elektromagnetischer Felder auf Expressionsmuster zu erhalten, wurde in den über PubMed und DIMDI zugänglichen, ab 1990 erschienenen medizinisch-wissenschaftlichen Publikationen recherchiert. Zur Auswertung berücksichtigt wurden kontrolliert durchgeführte Studien, bei denen insbesondere ein in der (nicht invasiven) Elektromagnetfeld-Therapie üblicherweise verwendetes niederfrequentes elektromagnetisches Signal verwendet wurde und in denen das Feld eindeutig beschrieben war (Tab. 1).

\section{Epidermaler Wachstumsfaktor}

Der Epidermale Wachstumsfaktor (EGF) stimuliert die Proliferation verschiedener Zelltypen. Eine Gruppe um VANESSA MANNI untersuchte am Instituto di Neurobiologia Medicina Molecolare in Rom den Einfluss eines niederfrequenten elektromagnetischen Feldes auf die Differenzierung humaner primärer Keratinozyten einer normalen Linie von Epithelzellen aus der oralen Mukosa von Kindern [13]. Unter dem Einfluss der 96 Stunden dauernden Exposition mit einem vorwiegend homogenen $2 \mathrm{mT}$ starken sinusförmigen 50-Hz-Elektromagnetfeld wurde weniger Zellwachstum und ein geringeres klonogenes Potenzial festgestellt. Die indirekte Immunfluoreszenz mit Fluoreszenz-Antikörper gegen Involucrin und $\beta$-Catenin wiesen dabei auf eine erhöhte Expres- 
Tab. 1. Kontrollierte Zellkultur-Studien zur Wirkung elektromagnetischer Felder auf Expressionsmuster von Wachstumsfaktoren ${ }^{*}$ elektrische Feldstärke)

\begin{tabular}{|c|c|c|c|c|c|c|c|c|c|}
\hline \multirow[t]{2}{*}{ Autor } & \multirow[t]{2}{*}{ Jahr } & \multirow[t]{2}{*}{ Methode } & \multirow[t]{2}{*}{ Spezies } & \multirow[t]{2}{*}{ Zellkultur } & \multicolumn{4}{|c|}{ Exposition } & \multirow[t]{2}{*}{ Ergebnis } \\
\hline & & & & & Frequenz & Feldstärke & Signalform & Dauer & \\
\hline Manni V & 2004 & In vitro & Mensch & Orale Keratinozyten & $50 \mathrm{~Hz}$ & $2 \mathrm{mT}$ & Sinus & $96 \mathrm{~h}$ & $\begin{array}{l}\text { Hemmung der EGF-Expression und } \\
\text { Einfluss auf Differenzierungs- } \\
\text { verhalten der Zellen. }\end{array}$ \\
\hline Vianale G & 2008 & In vitro & Mensch & $\begin{array}{l}\text { Keratinozyten } \\
\text { (HaCaT) }\end{array}$ & $50 \mathrm{~Hz}$ & $1 \mathrm{mT}$ & Sinus & $72 / 96 \mathrm{~h}$ & $\begin{array}{l}\text { Bildung von Chemokinen und } \\
\text { Wachstum der Keratinozyten } \\
\text { moduliert durch hemmenden Einfluss } \\
\text { auf NF-kB abhängigen Signalweg. }\end{array}$ \\
\hline Fitzsimmons RJ & 1992 & In vitro & Mensch & $\begin{array}{l}\text { Osteosarcoma Zellen } \\
\text { (TE-85) }\end{array}$ & $\begin{array}{l}10-16 \\
\text { pulse/s }\end{array}$ & $39 \mu \mathrm{T}$ & Sinus & $30 \mathrm{~min}$ & $\begin{array}{l}\text { Hinweis auf Beteiligung von IGF-II an } \\
\text { erhöhter Proliferation durch das } \\
\text { elektromagnetische Feld. }\end{array}$ \\
\hline Fitzsimmons RJ & 1995 & In vitro & Mensch & $\begin{array}{l}\text { Osteosarcoma Zellen } \\
\text { (TE-85) }\end{array}$ & $15,3 \mathrm{~Hz}$ & $\begin{array}{l}40 \mu \mathrm{T} \\
20 \mu \mathrm{T}\end{array}$ & $\begin{array}{l}\text { Sinus und } \\
\text { statisch }\end{array}$ & $10 \mathrm{~min}$ & $\begin{array}{l}\text { Stimulation der Freisetzung/Bildung } \\
\text { von IGF-II mit Stimulation der } \\
\text { Proliferation. }\end{array}$ \\
\hline Tepper OM & 2004 & $\begin{array}{l}\text { In vitro } \\
\text { in vivo }\end{array}$ & $\begin{array}{l}\text { Mensch } \\
\text { Maus }\end{array}$ & $\begin{array}{l}\text { Endothelzellen } \\
\text { (Nabelvene) } \\
\text { - }\end{array}$ & $\begin{array}{l}15 \text { pulse } / \mathrm{s} \\
15 \text { pulse } / \mathrm{s}\end{array}$ & $\begin{array}{l}1,2 \mathrm{mT} \\
1,2 \mathrm{mT}\end{array}$ & $\begin{array}{l}\text { Gepulste } \\
\text { asymmetrische } \\
\text { Signalform }\end{array}$ & $\begin{array}{l}8 \mathrm{~h} / \mathrm{d} \text { bis } 14 \mathrm{~d} \\
8 \mathrm{~h} / \mathrm{d} \text { bis } 14 \mathrm{~d}\end{array}$ & $\begin{array}{l}\text { Angiogenese erhöht durch stimulierte } \\
\text { endotheliale Freisetzung von FGF-2. } \\
\text { Mehr vaskuläre Einwachsungen. }\end{array}$ \\
\hline Longo FM & 1999 & In vivo & Ratte & - & 2 pulse/s & $0,3 \mathrm{mT}$ & Sägezahn, gepulst & $4 \mathrm{~h} / \mathrm{d}$ & $\begin{array}{l}\text { Hinweis auf Nerven-Regeneration } \\
\text { nach Verletzung durch verstärkten } \\
\text { Rückgang an NGF-Aktivität. }\end{array}$ \\
\hline Huang HM & 2006 & In vitro & Mensch & $\begin{array}{l}\text { Osteoblastenähnliche } \\
\text { Zellen (MG63) }\end{array}$ & - & $0,4 \mathrm{~T}$ & statisch & $12-72 \mathrm{~h}$ & Freisetzung von TGF- $\beta 1$ stimuliert. \\
\hline Zhuang H & 1997 & In vitro & Maus & $\begin{array}{l}\text { Osteoblastenähnliche } \\
\text { Zellen (MC3T3-E1) }\end{array}$ & $60 \mathrm{kHz}$ & $20 \mathrm{mv} / \mathrm{cm}^{*}$ & Sinus & $0,5-24 h$ & $\begin{array}{l}\text { Calcium/Calmodulin Signalweg an } \\
\text { TGF- } \beta 1 \text { mRNA-Induktion beteiligt. }\end{array}$ \\
\hline Nagai M & 1994 & In vivo & Huhn & - & 15 pulse $/ \mathrm{s}$ & $3,5 \mathrm{mT}$ & $\begin{array}{l}\text { Symmetrisches } \\
\text { Rechteck, gepulst }\end{array}$ & $0-19 d$ & $\begin{array}{l}\text { Osteoinduktiver Einfluss des EM } \\
\text { Feldes zumindest teilweise vermittelt } \\
\text { durch BMP-2 und BMP-4. }\end{array}$ \\
\hline Bodamyali T & 1998 & In vitro & Ratte & Osteoblasten & 15 pulse/s & $1,8 \mathrm{mT}$ & Sägezahn, gepulst & $6 \mathrm{~h}$ & $\begin{array}{l}\text { Mit der Expositionszeit korrelierte } \\
\text { Erhöhung der Gehalte an BMP-2 und } \\
\text { BMP-4 mRNA. }\end{array}$ \\
\hline Kafka WA & 2005 & In vitro & Mensch & Osteoblasten & 30 pulse/s & $100 \mu \mathrm{T}$ & $\begin{array}{l}\text { Sinus-modulierter } \\
\text { Exponentialanstieg, } \\
\text { gepulst }\end{array}$ & $5 \times 8 \mathrm{~h}$ & $\begin{array}{l}\text { Einfluss auf Proteinsynthese und } \\
\text { Aktivierung unterschiedlicher } \\
\text { molekulare Prozesse. }\end{array}$ \\
\hline
\end{tabular}

sion dieser Differenzierungs- und Adhäsionsmarker hin. Die nach 72 Stunden Exposition durchgeführte WesternBlot-Analyse bestätigte die erhöhte Differenzierungsrate durch die bei den exponierten Zellen weniger gefundenen EGF-Rezeptoren. Dieses Feld hemmte in normalen humanen Keratinozyten die Expression des Epidermalen Wachstumsfaktors und beeinflusste so das Differenzierungsverhalten dieser Zellen.

Immortalisierte humane Keratinozyten (HaCaT) dagegen zeigten hinsichtlich der Lebensfähigkeit der Zellen zwar ebenfalls keinen Unterschied zwischen den behandelten und nicht behandelten Zellen. Die Behandlung mit einem homogenen, $1 \mathrm{mT}$ starken sinusförmigen 50-Hz-Feld [14] führte jedoch hier nach 48 und 72 Stunden zu einer nicht signifikant höheren Wachstumsrate $(p<0,001)$ bei den behandelten Zellen. Bei ihrer Suche nach dem Einfluss eines elektromagnetischen Feldes mit extrem niederer Frequenz auf Chemokine stellten VIANALE et al. nach 72 Stunden Exposition darüber hinaus eine signifikant geringere Gen-Expression

\footnotetext{
2 Ein in der EMF-Therapie üblicher Übertragungsweg, bei welchem das elektromagnetische Feld übertragen wird welchem das elektromagnetische Feld ubertragen wird EMF-Quelle und Zielorgan ergebende elektrische Feld.
}

und Freisetzung der zur Familie der CC Chemokine zählenden Proteine CCL2 (MCP-1), CCL3 (MIP-1 $\alpha$ ) und CCL5 (RANTES) sowie des CXC Chemokins CXCL8 (IL-8) fest. Um zu erfahren, ob das elektromagnetische Feld dabei den Transkriptionsfaktor NF-кB beeinflusst, wurden die Gehalte an (aktiviertem) NF-кB p65 im Gesamtzellextrakt von proliferierenden exponierten und nicht exponierten Keratinozyten bestimmt. Bei einer negativen Korrelation mit der Anzahl Zellen zeigten die nicht exponierten Zellen über die Versuchszeit kontinuierlich abfallende Werte, wohingegen bei den behandelten Zellen bereits nach einer Stunde Exposition $\mathrm{NF}-\kappa \mathrm{B}$ fast nicht mehr nachzuweisen war. Insofern scheint dieses elektromagnetische Feld das Wachstum der Keratinozyten und die Bildung der Chemokine über eine Hemmung des NF-кBabhängigen Signalweges zu modulieren.

\section{Insulinähnlicher Wachstumsfaktor}

Wachstum und Überleben von Zellen wird allgemein durch den insulinähnlichen Wachstumsfaktor 2 (IGF-2) stimuliert. Zunächst hatte Fitzsimmons beobachtet, dass ein $50 \mathrm{~Hz}$ oszillierendes, kapazitiv gekoppeltes elektrisches Feld $^{2}$ von extrem geringer Amplitude und einer elektrischen Feldstärke im Kulturmedium von (geschätzten) 10-7 V/cm die Bildung des aus Hühnern gewonnenen embryonalen Knochengewebes erhöhte. Auch die von der Frequenz abhängige Proliferation von Knochenzellen schien von der im Kulturmedium erhöht freigesetzten mitogenen Aktivität abhängig zu sein.

Durch die Verwendung von Zellen aus der humanen Osteosarkom-Zelllinie TE-85 konnten weitere Erkenntnisse zur mitogenen Aktivität gewonnen werden [15]. TE-85-Zellen verhalten sich in Bezug auf die Bildung von Collagen Typ I, der Bildung von Osteolactin nach Behandlung mit 1,25(OH) Vitamin $\mathrm{D}_{3}$, sowie dem Vorhandensein von knochenspezifischer alkalischer Phosphatase und ihrer Reaktion auf eine Behandlung mit $1,25(\mathrm{OH})_{2}$ Vitamin $\mathrm{D}_{3}$ ähnlich wie normale humane Knochenzellen. Auch bei diesen Zellen zeigte sich eine Frequenzabhängigkeit. Nach 30 Minuten Exposition bei der Frequenz von $14 \mathrm{~Hz}$ war die Inkorporation von ${ }^{3} \mathrm{H}$-Thymidin mit $\mathrm{p}<0,1$ signifikant $27 \%$ höher. Die Veränderungen bei $10 \mathrm{~Hz}(-3 \%)$ und $12 \mathrm{~Hz}(+5 \%)$ waren nicht signifikant bzw. $0 \%$ bei $16 \mathrm{~Hz}$. Wurden die TE-85-Zellen 30 Minuten lang einem homogenen 14-Hz-Sinus- 
Feld mit einer elektrischen Feldstärke von $10^{-7} \mathrm{~V} / \mathrm{cm}$ - entsprechend einer magnetischen Feldstärke von in etwa $39 \mu \mathrm{T}$ - ausgesetzt und wurden danach, $\mathrm{zu}$ verschiedenen Zeiten, aus dem so konditionierten Medium Proben entnommen, war nach 60 Minuten beim Gehalt an IGF-2 ein deutliches Maximum erkennbar. Die Gehalte an IGF-1 lagen deutlich darunter und zeigten während dieser Zeit kein Maximum. Entsprechend der mRNA-Analyse hybridisierten mehrere Transkripte zu der IGF-2 cDNA-Probe mit deutlichem Anstieg der 4,8 und 7,0 Transkripte nach 30 Minuten Exposition durch das 14$\mathrm{Hz}$-Feld. Mit einer Steigerung um das 4,6-Fache über mehr als 24 Stunden war der Einfluss beim 4,8-kb-Transkript am deutlichsten. Das 7,0-kb-Transkript akkumulierte mit einem Maximalwert nach 3 Stunden und anschliessend kontinuierlich abfallenden Werten wesentlich geringer. IGF-1-Transkripte waren im Kulturmedium nicht nachweisbar.

Eine weitere Studie an derselben Zellkultur und Kombination aus einem gleichgerichteten, magnetischen (statischen) Feld in der Feldstärke von 20 $\mu \mathrm{T}$ mit einem $15,3 \mathrm{~Hz}$ sinusförmigen elektromagnetischen Wechselfeld mit einer (magnetischen) Spitzenfeldstärke von $40 \mu \mathrm{T}$ zeigte dessen Einfluss auf die Inkorporation von ${ }^{3} \mathrm{H}$-Thymidin in Abhängigkeit von der Zeit [16]. Die kurze Expositionszeit von 10 Minuten erhöhte einheitlich die Thymidin-Inkorporation. Da sich bei Expositionszeiten über 30 Minuten Unterschiede in den Werten um bis zu $200 \%$ zeigten, wurden die aus 7 unabhängigen Experimenten erhaltenen Werte gemittelt. Aufgrund des dann mit $\mathrm{p}<0,01$ festgestellten signifikanten Maximums wurde auf eine Expositionszeit von $10 \mathrm{~min}$ als optimal für die Inkorporation von Thymidin geschlossen. Der Einfluss durch das kombinierte magnetische/elektromagnetische Feld hinsichtlich einer höheren Zellproliferation und höheren Gehalten an IGF-2 erwies sich hier als

\footnotetext{
3 Asymmetrisches, mit einer Wiederholungsfrequenz von $15 \mathrm{~Hz}$ gepulstes elektromagnetisches Feld mit einer Pulsdauer von 4,5 msec und einer maximalen magnetischen Flussdichte von 1,2 mT (Anstiegszeit $200 \mu \mathrm{sec}$, Abfallzeit $25 \mu \mathrm{sec}$ )

4 Gepulstes Sägezahn-Signal mit einer Pulsdauer von 20 msec, $2 \mathrm{~Hz}$ Wiederholungs-Frequenz und maximaler magnetischer Feldstärke von 0,3 mT (Anstiegszeit 0,85 $\mathrm{msec}$, Abfallzeit 0,68 msec).
}

von der Zelldichte abhängig mit einer höheren stimulierenden Wirkung bei geringeren Zelldichten. So bestätigte sich die Hypothese, dass dieses elektromagnetische Feld die Freisetzung bzw. Bildung von IGF-2 in Knochenzellen stimuliert und ein erhöhter Gehalt an IGF-2 die Zellproliferation fördert.

\section{Fibroblasten-Wachstumsfaktor}

Fibroblasten-Wachstumsfaktoren (FGF), von denen bislang die Wachstumsfaktoren FGF-1 bis FGF-24 bekannt sind, stimulieren die Proliferation verschiedener Zelltypen, hemmen die Differenzierung bestimmter Vorläuferzellen und können während der Entwicklungsphase Gene zur Gewebedifferenzierung aktivieren.

Den Einfluss eines elektromagnetischen Feldes auf die Angiogenese untersuchte eine Arbeitsgruppe um TEPPER über die endotheliale Freisetzung von FGF-2 an einer Zellkultur von aus der menschlichen Nabelvene stammenden Endothelzellen [17]. Waren die auf einem Microcarrier wachsenden Zellen zwischen 7 und 10 Tagen dem elektromagnetischen Feld ${ }^{3}$ ausgesetzt, zeigten sich gegenüber den nicht behandelten Zellen deutliche Unterschiede im Wachstum der Tubuli. Exponierte Zellen bildeten 7 -mal mehr Tubuli mit ein oder zwei Microcarrier-Durchmesser, wobei Tubuli mit drei MicrocarrierDurchmesser und darüber nur bei den exponierten Zellen festzustellen waren. Nicht nur die Gesamtzahl der Tubuli pro Microcarrier war durch Exposition signifikant erhöht. Die Exposition hatte auch eine erhöhte Anzahl an Zellen und eine um den Faktor 3 stimulierte endotheliale Proliferation zur Folge. Ein Wachstum war jedoch nur bei den Endothelzellen $\mathrm{zu}$ beobachten und nicht bei den mit in die Untersuchung einbezogenen Osteoklasten und Fibroblasten. Die Kulturmedien bewirkten auch dann eine erhöhte Proliferation bei Endothelzellen, wenn diese selbst nicht dem elektromagnetischen Feld ausgesetzt worden waren. Die Autoren schlossen daraus auf einen löslichen Faktor und identifizierten aus mehreren Möglichkeiten mit einem immunologischen Nachweisverfahren (Enzyme Linked Immunosorbent Assay, ELISA) FGF-2 als ursächlichen Faktor. Vergli- chen mit den nicht exponierten Zellen wurde nach Exposition eine um den Faktor 5 höhere Bildung von FGF-2 gefunden. Die durch Northern-Blot-Analyse gefundene erhöhte Bildung von FGF-2 mRNA in den exponierten Zellen bestätigte diesen Befund. Selbst in vivo gelang der Nachweis einer Stimulation der Angiogenese durch ein elektromagnetisches Feld.

Matrigel, ein lösliches Membranmaterial, unterstützt bei subkutaner Injektion das Einwachsen von Gefässen. Wurde es transgenen Mäusen injiziert, welche danach mehrere Tage lang für jeweils 8 Stunden täglich in ihrem Käfig einem elektromagnetischen Feld ausgesetzt waren, war bei diesen Tieren ein signifikant grösseres Einwachsen von Gefässen festzustellen. Nach 3, 10 und 14 Tagen zeigten sich signifikant mehr vaskuläre Einwachsungen gegenüber den nicht behandelten Tieren mit einer Steigerung um mehr als das Doppelte bereits am dritten Tag. Mit ELISA wurde im exponierten Matrigel doppelt soviel an FGF-2 gefunden. Eine Veränderung bei den Wachstumsfaktoren TPO (Thrombopoietin), Ang-2 (Angiopoietin 2) und EGF konnte nicht festgestellt werden. Demnach beeinflusste das Signal dieses pulsierenden elektromagnetischen Feldes die Angiogenese primär über die Stimulation einer endothelialen Freisetzung von FGF-2 und induzierte parakrine und autokrine Veränderungen im benachbarten Gewebe.

\section{Nerven-Wachstumsfaktor}

Zusammen mit Kollegen aus verschiedenen amerikanischen Universitäten prüfte LONGO bereits Ende der 90erJahre den Einfluss eines extrem niederfrequenten, pulsierenden elektromagnetischen Sägezahn-Signals ${ }^{4}$ auf den Nerven-Wachstumsfaktor (NGF) selbst - d.h. das Protein - und dessen Aktivität in einem verletzten Nerven und/oder im Spinalganglion während der ersten 6 bis 72 Stunden der Regeneration [18]. Nach Durchtrennung des Nervus ischiadicus von Ratten im mittleren Bereich des rechten Femur wurden die Tiere der Verum-Gruppe verschiedene Tage lang 4 Stunden täglich dem elektromagnetischen Feld ausgesetzt und die NGF-Aktivität im Spinalganglion (NGF-ähnliche Aktivität), in 
5-mm-Nervensegmenten proximal und distal zur Verletzung und in den entsprechenden 5-mm-Segmenten auf der anderen Körperseite bestimmt.

Dieses elektromagnetische Feld führte insgesamt $\mathrm{zu}$ einer signifikanten $\mathrm{Ab}$ nahme $(p<0,02)$ an NGF-ähnlicher Aktivität im Nervengewebe mit deutlichen Abnahmen im proximalen, distalen und kontralateralen, nicht operierten Nerv.

Bei den Nervensegmenten zeigte sich bei den exponierten, nicht operierten kollateralen Nerven nach 6 Stunden eine Abnahme an NGF-ähnlicher Aktivität um $61 \%$. Nach 24 Stunden betrug die Abnahme noch $26 \%$ und war nach 72 Stunden um 14\% höher als bei den zum Schein behandelten, nicht operierten, kollateralen Nervensegmenten. Bei den exponierten proximalen Nervensegmenten war die Aktivität im Vergleich zu den zum Schein behandelten Tieren nach 6 Stunden um 13\% erhöht, jedoch nach 24 Stunden um $13 \%$, nach 48 Stunden um $27 \%$ und nach 72 Stunden um 25\% geringer. Bei den distalen Nervensegmenten führte die Exposition nach 6 Stunden zu einer Abnahme an Aktivität um 52\%. 24 Stunden danach war sie um $33 \%$, nach 48 Stunden um $27 \%$ und zu Versuchsende nach 72 Stunden um $36 \%$ geringer als bei der Scheinbehandlung.

Bei dem auf der gleichen Seite zur Durchtrennung liegenden Spinalganglion wurde bei den zum Schein behandelten Tieren zwischen 6 und 24 Stunden nach Durchtrennung eine um 50\% höhere NGF-ähnliche Aktivität festgestellt, während sie bei den exponierten Tieren nur um 6\% erhöht war. Dieser leichte Anstieg an NGF-ähnlicher Aktivität deckte sich mit dem durch das elektromagnetische Feld induzierten Abfall an Aktivität im Nervengewebe, zeigte jedoch keine statistische Signifikanz. In dem der Durchtrennung gegenüber liegenden Spinalganglion hingegen war

\footnotetext{
5 Kapazitiv gekoppeltes 60 kHz Sinus-Signal mit 20 mV/cm elektr. Feldstärke.

6 N-(6-Aminohexyl)-5-Chloro-1-Naphtalensulfonamid (CAS-Nr. 65595-90-6).

7 Elektromagnetisches Wechselfeld mit symmetrischem Rechtecksignal (3,5 mT magnetische Flussdichte / $9 \mathrm{mV}$ m elektrische Feldstärke), Pulsdauer $200 \mu \mathrm{sec}$ bzw. 24 usec. Die einzelnen Signalfolgen (Bursts) dauerten $5 \mathrm{msec}$ bei einer Wiederholungsfrequenz von $15 \mathrm{~Hz}$.

8 Elektromagnetisches Feld mit Sägezahn-Charakteristik und einer maximalen magnetischen Flussdichte von 1,8 mT, einer Signaldauer von 225 usec und Wiederho$1,8 \mathrm{mT}$, einer Signaldauer von $225 \mu \mathrm{sec}$ und Wiederho4t,5 ms dauernden Signalfolgen (Bursts) von $15 \mathrm{~Hz}$
}

bei den scheinbehandelten und bei den exponierten Tieren eine Aktivitätserhöhung um $100 \%$ feststellbar.

ELISA und die Verwendung von NGFspezifischen Antikörpern bestätigten mit $p<0,0001$ den signifikant geringeren Gehalt an NGF im exponierten Nervengewebe. Bei exponierten unverletzten Tieren wurden weder im Spinalganglion, noch im Nervengewebe geringere Werte gefunden. Nach Meinung der Autoren fördert das elektromagnetische Feld möglicherweise die Regeneration von Nerven unmittelbar nach der Verletzung durch eine Verstärkung des Rückgangs an NGF-Aktivität.

\section{Transformierender \\ Wachstumsfaktor Beta}

Der zu den Rezeptor-Serin-/Threoninkinasen gehörende Transformierende Wachstumsfaktor Beta (TGF- $\beta$ ) gehört $\mathrm{zu}$ einer TGF- $\beta$-Superfamilie mit lokaler Vermittlung bei der Steuerung vieler biologischer Funktionen. Osteoblasten-ähnliche MG63-Zellen - über 12, 24, 48 und 72 Stunden kontinuierlich einem 0,4 T starken statischen Magnetfeld ausgesetzt - setzten mehr TGF$\beta 1$ frei. Verglichen mit den scheinexponierten Zellen setzten die exponierten Zellen eine mit $\mathrm{p}<0,05$ statistisch signifikant höhere Menge an TGF$\beta 1$ frei. Der Wachstumsfaktor wurde sofort mit Beginn der Exposition freigesetzt und während der gesamten Zeit der Kultivierung laufend herunter geregelt. Noch nach 12 Stunden lag der Gehalt an TGF- $\beta 1$ in den exponierten Zellen um den Faktor 1,7 höher und blieb dann über die restliche Zeit im Mittel, statistisch signifikant, um den Faktor 1,5 über den Werten der scheinbehandelten MG63-Zellen [19].

ZHUANG et al. setzten MC3T3-E1 Osteoblastenzellen 24 Stunden lang einem elektromagnetischen Feld ${ }^{5}$ aus. Die Bestimmung des Gehalts an TGF- $\beta 1 \mathrm{mRNA}$ bestätigte die mit dem statischen Magnetfeld gefundene erhöhte Proliferation und gibt einen Hinweis auf eine dabei mögliche Beteiligung des Calcium/Calmodulin Übertragungsweges.

Nach 24 Stunden zeigten die behandelten Zellen mit $\mathrm{p}<0,05$ eine um 38,8\% signifikant höhere TGF- $\beta 1$ Aktivität als die zur Kontrolle dienenden Zellen. Der bereits ab 2 Stunden Exposition durch
PCR festgestellte, signifikant erhöhte Gehalt an mRNA belegt für das hier verwendete Zellsystem einen direkten Zusammenhang zwischen der (erhöhten) TGF- $\beta 1$-Aktivität und dem höheren Gehalten an TGF- $\beta 1$ mRNA. Verapamil und der, chemisch vom Naphthalin sich ableitende, Calmodulin-Antagonist $\mathrm{W}^{6}$ hemmten die durch das elektromagnetische Feld induzierte Osteoblastenproliferation, ohne das Zellwachstum unbehandelter Osteoblasten zu beeinflussen. Dieses deutet darauf hin, dass eine Calcium-/Calmodulin-abhängige Proteinkinase an dem durch elektromagnetische Stimulation in den Osteoblasten induzierten höheren Gehalt an TGF- $\beta 1$ mRNA beteiligt ist [20].

\section{Knochenmorphogenese-Proteine}

Hinsichtlich der möglichen Wirkung eines Magnetfeldes auf die zur TGF- $\beta$ Untergruppe zählenden Knochen-Morphogenese-Proteine (BMP) wurde bereits 1994 anhand von Untersuchungen mit embryonalem Gewebe aus dem Schädeldach von Hühnern eine erhöhte Expression beim BMP-2 und BMP-4 nachgewiesen. Bei den ab dem Embryonalstadium 19 Tage lang einem symmetrischen Wechselfeld ${ }^{7}$ ausgesetzten Eiern war im Vergleich zu den nicht behandelten Eiern allerdings nur bei Zellen aus jüngeren Embryonen im Alter zwischen 15 und 17 Tagen ein statistisch signifikanter Einfluss feststellbar. Die ausgeprägtere, erhöhte Expression vom BMP-2 und BMP-4 mRNA bei Zellen jüngerer Embryonen weist hin auf eine zumindest teilweise Vermittlung von BMP-2 und BMP-4 beim Einfluss eines elektromagnetischen Feldes auf Knochenzellen [21]. Auch mit einem anderen elektromagnetischen Feld ${ }^{8}$ ist bei Osteoblasten aus dem Schädeldach von 3 Tage alten Ratten eine erhöhte Expression beschrieben. Ausser erhöhten Gehalten an BMP-2 und BMP-4 zeigte sich hier eine zeitliche Abhängigkeit zur Behandlungsdauer. Nach 30 Minuten Behandlung erreichte der Gehalt an BMP-4 mRNA mit einer Steigerung von 29,6\% ein Maximum und fiel nach weiteren 30 Minuten auf 21\% zurück. Bei der BMP-2 mRNA dagegen stiegen die absoluten Gehalte auf bis zu $49,4 \%$ bis zum Ende der Exposition von 60 Mi- 
nuten kontinuierlich an. Die Gehalte an beiden mRNAs waren dabei deutlich höher in Zellen, welche kontinuierlich 30 Minuten dem elektromagnetischen Feld ausgesetzt waren und nicht 15 Minuten ohne Exposition weiter inkubiert wurden. Demzufolge führte der Wegfall des elektromagnetischen Feldes nach der Hälfte der Inkubationszeit zu einer Down-Regulation bei der BMP-2und -4-Transkription und lässt auf eine zeitliche Abhängigkeit zur Expositionszeit auch bei einer nur kurzen Behandlungsdauer schliessen [22].

\section{Osteoblasten-Proliferation und allgemeine Aktivierung molekularer Prozesse}

Ein breiter Einfluss eines elektromagnetischen Feldes auf die Proteinsynthese geht aus der Stimulation humaner Osteoblasten mit einem 18-mal schwächeren, speziell pulsierenden elektromagnetischen Signal $^{9}$ hervor [23]. Gegenüber der Kontrolle ergab sich bereits nach kurzer Zeit eine signifikante, mit zunehmender Flussdichte korrelierte Steigerung der Proliferationsraten um bis zum Fünffachen im Bereich der maximalen mittleren Flussdichte. Eine daraufhin mit den Proben im Bereich der maximalen Flussdichten durchgeführte GenChip-Analyse belegte signifikante Veränderungen simultan bei der Up- und Down-Regulation von Genprodukten. So wurde beispielsweise bei dem Ring-FingerProtein Typ 6 (RNF6) und dem Fettsäuren-Bindungsprotein Typ 4 (FABP4) eine Up-Regulation beobachtet, während gleichzeitig beim TNPO2 (Transportin 2) und ARH1 (ras homolog gene family member 1) eine Down-Regulation festzustellen war. Das hier verwendete elektromagnetische Signal beeinflusste offensichtlich nicht nur die Proteinsynthese auf genetischem Niveau. Es aktivierte dabei auch völlig unterschiedliche molekulare Prozesse.

\footnotetext{
9 Elektromagnetisches Signal mit einer Wiederholungs rate von $30 \mathrm{~Hz}$ und maximaler magentischer Flussdichte von $100 \mu \mathrm{T}$. Von Null beginnend, steigt seine Intensität zunächst an und fällt danach wieder auf einen Wert ab, der jedoch höher als der Ausgangswert liegt. Die Abfolge dieser Intensitätswechsel wird innerhalb des $30 \mathrm{msec}$ dauernden Signals mit zunehmender Zeit des Flanken des An und Abstiegs.
}

\section{Schlussfolgerungen}

Die wenigen vorhandenen, hier dargestellten Studien belegen eine auf molekularer Ebene äusserst adaptive, vielseitige und individuelle Wirkung elektromagnetischer Signale auf die Expression von Wachstumsfaktoren. Daraus jedoch bereits wissenschaftlich belegte Aussagen hinsichtlich ihrer therapeutischen Anwendbarkeit zu treffen, wird wegen der vielen unterschiedlichen Signale noch viele Jahre Forschung in Anspruch nehmen. Noch sind diese Ergebnisse nichts weiter als ein kleiner Mosaikstein im grossen Puzzle der Elektromagnetfeldtherapie.

Der Verkauf von Elektromagnetfeldsystemen ist für die Unternehmen ein bekanntermassen profitables Geschäft und sie sollten, nicht zuletzt in ihrem eigenen Interesse, zur Förderung medizinisch-wissenschaftlicher Erkenntnisse beitragen. Gemäss ihren Äusserungen ist dieses auch der Fall. Bei genauerem Hinsehen entpuppt sich ihr starkes Interesse an Wissenschaft und Forschung allerdings allzu oft als ein nur dem Marketing dienendes Argument mit dem Ziel, die zur Wirksamkeit der Produkte vorgelegten Dokumente seriöser erscheinen zu lassen.

Insofern bleibt $\mathrm{zu}$ hoffen, dass sich künftig von Herstellern und Vertreibern unabhängige, wissenschaftliche Institutionen stärker als bisher mit den gesundheitlichen Wirkungen elektromagnetischer Felder befassen. Vor allem, um auf diesem Gebiet nicht einschlägig bewanderten Angehörigen medizinischer Fachkreise fundierte Kenntnisse zur Verfügung zu stellen, um künftig besser die Spreu vom Weizen trennen zu können.

\section{Literatur}

1. Jelinek R, Bláha J, Dbalý J: persönliche Mitteilung. 2002: Karls-Universität, Prag.

2. Goodman R, Blank M: Insights into electromag netic interaction mechanisms. J Cell Physio 2002;192(1):16-22.

3. Markov MS: How to go for magnetic field therapy? Biological Effects of Electromagnetic Fields, 2nd Int. Workshop, 7.-11. October 2002 [cited 2008 18. Juli]; available from: http://www.bioingenieria.edu.ar/academica/catedras/radia ciones/mft.pdf.

4. Liboff AR: Signal Shapes in Electromagnetic Therapies: A Primer, in Bioelectromagnetic Medicine, P.J. Paul J. Rosch and M.S. Markov, Editors. 2004, Informa Healthcare. p. 880.

5. Blank M, Goodman R: Initial interactions in electromagnetic field-induced biosynthesis. J Cell Physiol 2004;199(3):359-63.
6. Pieber K, Schuhfried O, Fialka-Moser V: [Pulsed electromagnetic fields (PEMF) - results in evidence based medicine]. Wien Med Wochenschr 2007:157(1-2):34-6.

7. Quittan $M$ et al.: [Clinical effectiveness of magnetic field therapy - a review of the literature]. Acta Med Austriaca 2000:27(3):61-8.

8. Glaser R: Elektro-Magneto-Therapie - Situation und Perspektiven. News Letter Forschung 2004;1 16-25.

9. McKay JC, Prato FS, Thomas AW: A literature review: The effects of magnetic field exposure on blood flow and blood vessels in the microvasculature. Bioelectromagnetics 2007;28(2):81-98.

10. Schuhfried O. et al.: The effects of low-dosed and high-dosed low-frequency electromagnetic fields on microcirculation and skin temperature in healthy subjects. Int J Sports Med 2005;26(10):886-90.

11. Funk RH, Monsees TK: Effects of electromagnetic fields on cells: physiological and therapeutical approaches and molecular mechanisms of interaction. A review. Cells Tissues Organs 2006; 182(2):59-78.

12. Gartzke J, Lange K: Cellular target of weak magnetic fields: ionic conduction along actin filaments of microvilli. Am J Physiol Cell Physiol 2002; 283(5):C1333-46.

13. Manni $V$ et al.: Low electromagnetic field $(50 \mathrm{~Hz})$ induces differentiation on primary human oral keratinocytes (HOK). Bioelectromagnetics 2004;25(2): 118-26.

14. Vianale $\mathrm{G}$ et al.: Extremely low frequency electromagnetic field enhances human keratinocyte cell growth and decreases proinflammatory chemokine production. $\mathrm{Br} J$ Dermatol 2008;158(6): 1189-96.

15. Fitzsimmons RJ et al: Low-amplitude, low-frequency electric field-stimulated bone cell proliferation may in part be mediated by increased IGF-II release. J Cell Physiol 1992; 150(1):84-9.

16. Fitzsimmons RJ et al.: Combined magnetic fields increase insulin-like growth factor-II in TE-85 human osteosarcoma bone cell cultures. Endocrinology 1995;136(7):3100-6.

17. Tepper $\mathrm{OM}$ et al.: Electromagnetic fields increase in vitro and in vivo angiogenesis through endothelial release of FGF-2. FASEB J 2004:18(11): 1231-3.

18. Longo FM et al.: Electromagnetic fields influence NGF activity and levels following sciatic nerve transection. J Neurosci Res 1999;55(2):230-7.

19. Huang HM et al.: Static magnetic fields up-regulate osteoblast maturity by affecting local differentiation factors. Clin Orthop Relat Res 2006; 447:201-8

20. Zhuang $\mathrm{H}$ et al.: Electrical stimulation induces the level of TGF-beta1 mRNA in osteoblastic cells by a mechanism involving calcium/calmodulin pathway. Biochem Biophys Res Commun 1997: 237(2):225-9.

21. Nagai $M$, Ota M: Pulsating electromagnetic field stimulates mRNA expression of bone morphogenetic protein-2 and -4. J Dent Res 1994;73(10): 1601-5.

22. Bodamyali T et al.: Pulsed electromagnetic fields simultaneously induce osteogenesis and upregulate transcription of bone morphogenetic proteins 2 and 4 in rat osteoblasts in vitro. Biochem Biophys Res Commun 1998;250(2):458-61.

23. Kafka WA, Schütze N, Walther M: Einsatz extrem niederfrequent (BEMER-typisch) gepulster schwacher elektromagnetischer Felder im Bereich der Orthopädie. Orthopädische Praxis 2005;41(1):23-25.

\section{Disclosure Statement}

The author declares that no financial or other conflict of interest exists in relation to the content of this article.

\section{Korrespondenzadresse:}

Dr. rer. nat. Gerhard W. Ruoff Briggla 9, CH-9475 Sevelen

gerhard.ruoff@supra.net 